This item was submitted to Loughborough's Research Repository by the author.

Items in Figshare are protected by copyright, with all rights reserved, unless otherwise indicated.

\title{
Printed, fully metal oxide, capacitive humidity sensors using conductive indium tin oxide inks
}

PLEASE CITE THE PUBLISHED VERSION

https://doi.org/10.1021/acsaelm.0c00660

\section{PUBLISHER}

American Chemical Society (ACS)

\section{VERSION}

VoR (Version of Record)

\section{PUBLISHER STATEMENT}

This is an Open Access Article. It is published by American Chemical Society under the Creative Commons Attribution 4.0 Unported Licence (CC BY). Full details of this licence are available at: http://creativecommons.org/licenses/by/4.0/

\section{LICENCE}

CC BY 4.0

\section{REPOSITORY RECORD}

McGhee, Jack, Jagdeep Sagu, Darren Southee, Peter S.A Evans, and Upul Wijayantha-Kahagala-Gamage. 2020. "Printed, Fully Metal Oxide, Capacitive Humidity Sensors Using Conductive Indium Tin Oxide Inks". Loughborough University. https://hdl.handle.net/2134/13347494.v1. 


\title{
Printed, Fully Metal Oxide, Capacitive Humidity Sensors Using Conductive Indium Tin Oxide Inks
}

\author{
Jack R. McGhee,* Jagdeep S. Sagu, Darren J. Southee, Peter. S. A. Evans, and K. G. Upul Wijayantha
}

Cite This: ACS Appl. Electron. Mater. 2020, 2, 3593-3600

Read Online

ABSTRACT: In this research, fully metal oxide, capacitive humidity sensors for printed electronic applications have been designed and fabricated through the development of conductive indium tin oxide and dielectric aluminum oxide inks for the screen-printing process. Sensors were printed in a parallel plate configuration in 4 and $9 \mathrm{~cm}^{2}$ conductive plate areas. Typically, commercially available discrete humidity sensors have a sensitivity in the range of $0.2-0.5 \mathrm{pF}$ / $\mathrm{RH} \%$, whereas the printed humidity sensors presented in this paper have sensitivities of $0.85-7.76 \mathrm{pF} / \mathrm{RH} \%$ depending on the sensor size, allowing for customizable properties. Response times were measured using a weighted average and found to be $21.4 \mathrm{~s}$ on average and recovery times were $4.8 \mathrm{~s}$ on average. The sensing performance was highly linear $\left(R^{2}>0.97\right)$ for sensors of all sizes across the measured humidity range of 5-95\%. Impedance spectroscopy was used to determine the sensing mechanism, and the mechanism was simulated and
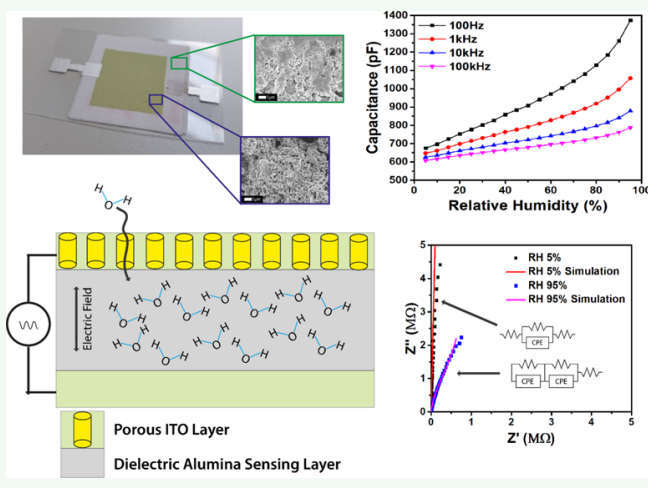
matched with experimental data. The sensing mechanism analysis shows that the sensing is primarily dictated by alumina at a lower relative humidity. The ITO contributes through increasing the ionic conductivity at a higher relative humidity, contributing to the high linearity of the sensor.

KEYWORDS: screen printing, humidity sensors, ink formulation, printed and flexible electronics, parallel plate capacitors

\section{INTRODUCTION}

Humidity sensing is a crucial environmental monitoring method for many industrial sectors, including agriculture, medicine, meteorology, and environmental health. ${ }^{1,2}$ In recent years, as developments have been made with Internet of Things (IoT) technology, humidity sensors have been used as a key component in developing smart monitoring systems. ${ }^{3}$ Examples of these include smart farming, storage monitoring, and home automation. ${ }^{4-6}$ Simultaneously, printed electronics has become a popular choice of the manufacturing method, developing smart packaging through the use of IoT and sensors. $^{7-10}$

Fabricating sensors through printing provides benefits such as high throughput and low-cost manufacturing with success being found in mechanical sensing. ${ }^{11,12}$ These sensors typically use printed silver or carbon. ${ }^{13}$ This is especially true for flexible electronics where humidity sensors are typically made from polymer composites ${ }^{14}$ or carbon allotropes ${ }^{15}$ or composites. ${ }^{16}$ However, environmental monitoring such as temperature or humidity sensing requires materials that are yet to be properly integrated into printed manufacturing methods. Metal oxides are good candidates for this; conductive metal oxides are typically more stable in the environment than metals. They also have a long history of gas and humidity sensing. ${ }^{17}$

Two key metal oxides are aluminum oxide, a common ceramic used in traditionally manufactured humidity sensors, ${ }^{18}$ and indium tin oxide, a conductive metal oxide material which has also seen use in humidity and gas sensing. ${ }^{19}$ Previous work has shown that the two materials can be combined as a printed conductive/dielectric parallel plate capacitor. ${ }^{20}$ However, the printed variants of these materials have never been researched as humidity sensors.

In this paper, and for the first time, indium tin oxide (ITO) and aluminum oxide $\left(\mathrm{Al}_{2} \mathrm{O}_{3}\right)$ inks are formulated and used to manufacture capacitive metal oxide humidity sensors through screen printing. Sensors were printed in a parallel plate configuration with interconnect for the electrodes printed in silver. The sensors were found to be able to sense the relative humidity between 5 and $95 \%$ and displayed a high degree of linearity $\left(R^{2}>0.97\right)$ for the entire range. Both the sensitivity alongside the response and recovery times changed depending on the sensor size, allowing for customizable properties. Smaller sensors were found to have fast response times, whereas larger sensors benefited from high sensitivity. The sensing mechanism of the sensor was analyzed using

Received: July 30, 2020

Accepted: October 11, 2020

Published: October 22, 2020 


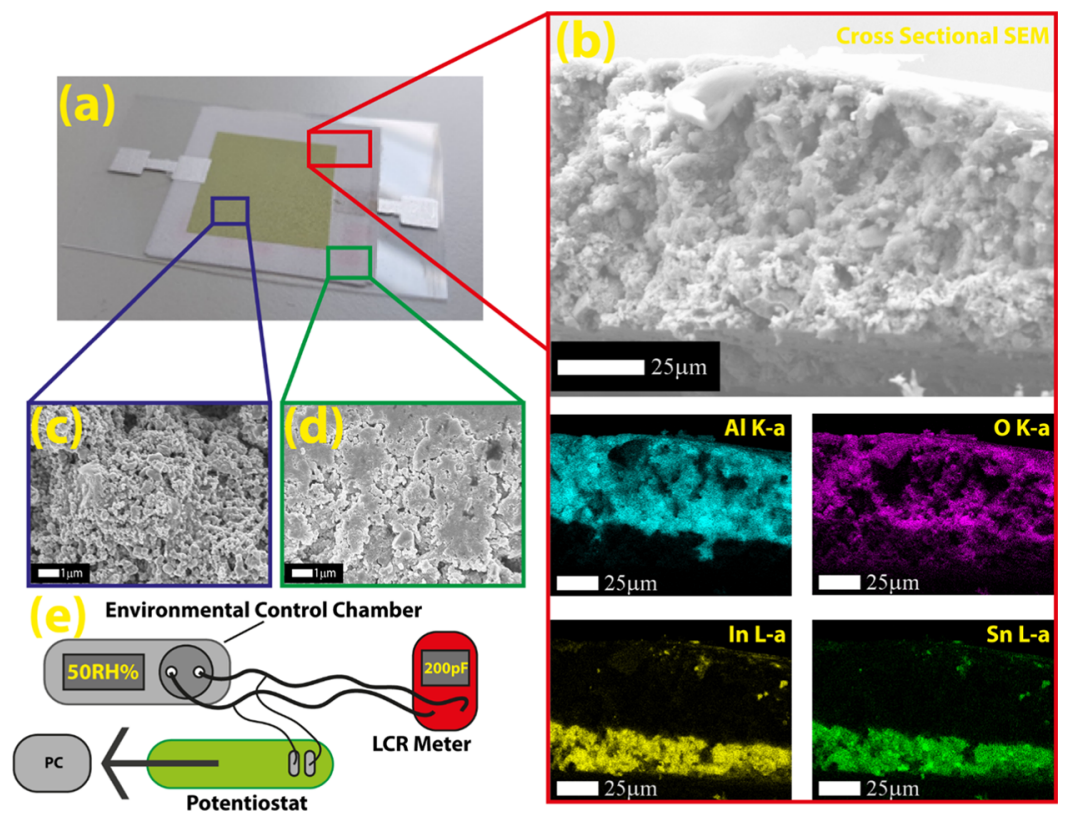

Figure 1. Image of a (a) $4 \mathrm{~cm}^{2}$ fully printed capacitive humidity sensor with the red image highlight detailing (b) a cross-sectional SEM/EDX of the ITO/alumina bilayer sensing structure and EDX images displaying Al K-a, O K-a, In L-a, and Sn L-a layers with scale bars indicating $50 \mu \mathrm{m}$; the blue highlight details (c) a top-down SEM of the printed conductive ITO layer at $25000 \times$ magnification and the green highlight shows (d) a topdown SEM of the alumina dielectric printed layer; finally, (e) demonstrates the experimental setup used for this research, detailing the sensor being placed in an environmental chamber with measurements being performed on an LCR meter and potentiostat.

impedance spectroscopy, and it was found to use both the indium tin oxide and aluminum oxide layers to allow for sensing across the full relative humidity range. Overall, in this study, a fully metal oxide, capacitive humidity sensor fully printed onto a flexible substrate has been demonstrated. This provides good sensitivity, good response and fast recovery times alongside high stability, and the option for customizable properties through size alteration.

\section{EXPERIMENTAL METHODS}

2.1. Ink Materials and Formulation. The materials used to fabricate the sensors are characterized and detailed in previously published works. ${ }^{20,21}$ Electrically conducting metal oxide inks were developed using indium tin oxide powder (Alfa Aesar, 99.9\% Metals Basis) dispersed into a nitrocellulose-based vehicle. Dielectric metal oxide inks were developed using aluminum oxide $(<50 \mathrm{~nm}$ nanopowder, Sigma Aldrich) powder again mixed into a nitrocellulose vehicle. Metallic interconnects were printed using Gwent Electronic Material Flexible Silver Ink (C2131014D3). Inks were formulated and dispersed via a mechanical mill processing using an EXAKT $50 \mathrm{~L}$ three roll mill. Powders were mixed with the vehicle and milled for 10 min at a time with a roller gap set to $1 \mu \mathrm{m}$. The ink was then collected and placed into a container ready for screen printing. The sheet resistance of the low-temperature oven-cured conductive ITO ink is on average $57.77 \Omega /$ sq measured via four-point probe.

2.2. Sensor Fabrication. The design of the sensors is again detailed in a previously published work. ${ }^{20}$ The fully printed sensor can be seen in Figure 1a. Interconnects were first printed using the silver ink and cured. A square of ITO ink was then printed and cured followed by three printed layers of alumina. A second ITO conductive plate was then printed on top of the dielectric alumina layer followed by a second silver contact. All curing steps were performed for $10 \mathrm{~min}$ at $110{ }^{\circ} \mathrm{C}$ in a ventilated box oven. The structure of the ITO ink is shown in Figure 1c, which shows a porous layer with a fully conducting network of ITO particles. The substrate used clear Melinex ST504 heat-stabilized poly(ethylene terephthalate) (PET) and was donated by Dupont Teijin Films. After each printing step, the ink was cured for $10 \mathrm{~min}$ at $110^{\circ} \mathrm{C}$. Screen printing was performed on a DEK 1202 using stainless steel screens with 230 (75/36) meshes, each providing a $13 \mu \mathrm{m}$ emulsion (wet film) thickness upon printing. The off contact height, which is also known as the distance between the screen and the printing medium, was set at $1 \mathrm{~mm}$. The deflection angle of the printing squeegee was set at an angle of $60^{\circ}$. The DEK 1202 screen printer has a graduated knob for setting the squeegee pressure, with the pressure being set at a value of 2 on this knob.

In printed electronics for dielectric layers, pinholes represent a challenge. It was found that by printing three layers of dielectric material, pinholes could be preventing a short circuit between the two conductive plates in the capacitor and enabled an optimized dielectric constant $\left(\varepsilon_{\mathrm{r}}\right)$ of $\varepsilon_{\mathrm{r}}=7.6$ and a loss tangent $(\tan \delta)$ of $\tan \delta=0.05$ at $100 \mathrm{~Hz}$. Cross-sectional SEM/EDX was performed to ensure good separation of layers between the ITO and alumina and can be seen in Figure $1 \mathrm{~b}$. As illustrated, the single layer of indium and tin is well defined and separated from the aluminum layer, allowing for enough separation to prevent conducting materials to pass through the alumina and short out the capacitive structure. The permittivity is slightly lower, and the loss tangent is higher than that of fully sintered ceramic alumina (typically $\varepsilon_{\mathrm{r}}=9.8$ and $\tan \delta=0.0001$ at low frequencies). This is due to the porous nature of the printed ink as seen in Figure 1d creating air voids in the material, which is known to decrease permittivity. ${ }^{22}$ The increase in electric field loss can be attributed to the leftover organics in the printed film, which are typically more lossy than ceramic materials. However, these values are excellent for low-frequency use, of which humidity sensors are typically used in.

2.3. Measurement Equipment. Capacitance measurements were taken using a UNI-T digital LCR meter (UT612); these measurements were taken using the LCR meter connected to a PC using the UNI-T digital multimeter software provided. Measurements of capacitance were taken with the LCR meter with varying frequencies of $100 \mathrm{~Hz}, 1 \mathrm{kHz}, 10 \mathrm{kHz}$, and $100 \mathrm{kHz}$; the default setting of the LCR meter is $1 \mathrm{kHz}$. Humidity measurements were taken using a Rotronic Instruments HygroGen humidity and temperature measurement chamber. The software controller for the chamber was the Eurotherm 2704 temperature and humidity controller. The reference humidity sensing probe used in this research is the Rotronic Instruments HygroClip S control probe, which has been combined 
(a)

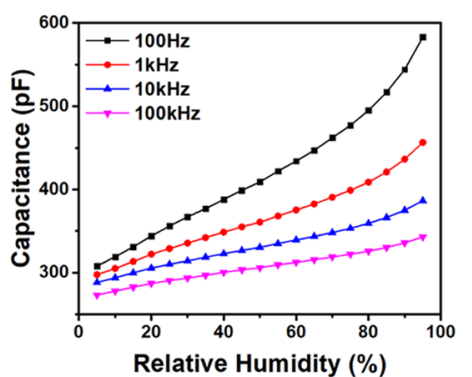

(c)

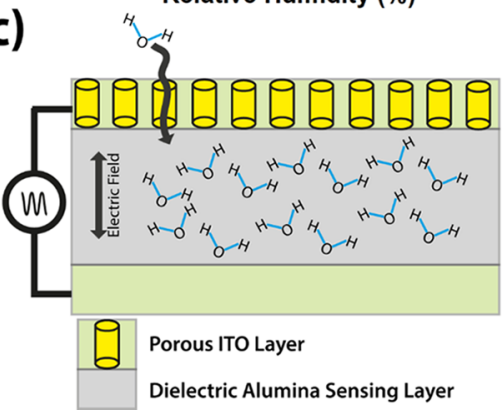

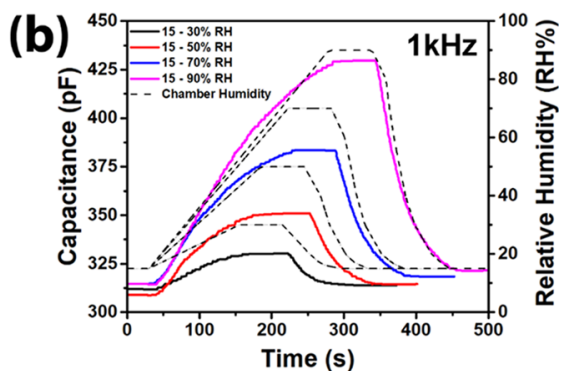

(d)

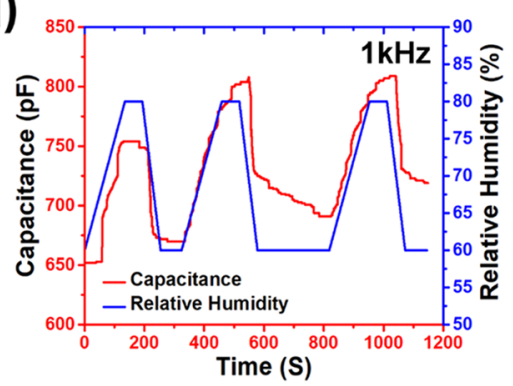

Figure 2. Graph detailing a (a) $4 \mathrm{~cm}^{2}$ fully printed capacitive humidity sensor's humidity sensing characteristics of relative humidity versus capacitance with the corresponding $9 \mathrm{~cm}^{2}$ graph found in Figure S1; (b) humidity response characteristics for a $4 \mathrm{~cm}^{2}$ humidity printed metal oxide humidity detailing the capacitance and relative humidity change versus time with the corresponding $9 \mathrm{~cm}^{2}$ graph found in Figure S2; (c) a schematic detailing the parallel plate capacitive sensor structure and dielectric layer water absorption; and (d) the dynamic response of humidity sensors showing sensor response versus multiple changes in relative humidity over time for a $4 \mathrm{~cm}^{2}$ sensor.

with a PT100 temperature sensing probe to provide both reference humidity and temperature measurements. Impedance spectroscopy measurements were taken using a Metrohm Autolab Instruments multichannel potentiostat/galvanostat (PGSTAT12) using Metrohm's Frequency Response Analyzer software (v4.7.900). The general experimental setup can be seen in Figure 1e. Data analysis of complex impedance data was performed using Autolab Nova (v2.0). Impedance measurements were taken using 50 measuring steps between $100 \mathrm{kHz}$ and $0.1 \mathrm{~Hz}$ with an amplitude of $0.01 \mathrm{~V}$. For the calculation of the material layer thicknesses, stylus profilometry was used, the profilometer was a Talysurf CLI 2000, and data was analyzed in the Talymap Platinum (v5) software package. Scanning electron microscopy images were taken on a Jeol $7800 \mathrm{~F}$ field emission gun scanning electron microscope (FEG-SEM). The accelerating voltage used for all SEM images was $5 \mathrm{kV}$, using a typical working distance of $10 \mathrm{~mm}$. Energy-dispersive X-ray spectroscopy (EDX/EDS) measurements were taken with the same Jeol $7800 \mathrm{~F}$ FEGSEM at $20 \mathrm{kV}$, working distance was changed to 5 $\mathrm{mm}$, and software processing was performed on Oxford Instruments AZTEC. Sheet resistance measurements were taken using a Jandel HM20 four-point probe.

\section{RESULTS AND DISCUSSION}

3.1. Humidity Sensing Characteristics. Capacitance versus relative humidity was measured at four different frequencies for both 4 and $9 \mathrm{~cm}^{2}$ sensor structures, $100 \mathrm{~Hz}$, $1 \mathrm{kHz}, 10 \mathrm{kHz}$, and $100 \mathrm{kHz}$, as shown in Figure 2 for three replicates. Capacitance increases with an increase in relative humidity for all frequencies as expected due to water absorption altering the dielectric properties of the alumina. The sensors were measured between 5 and 95\% relative humidity, the limits of the environmental chamber used, with temperature kept constant at $25{ }^{\circ} \mathrm{C}$. The average sensitivity of the sensors and the standard errors of the mean were calculated for each measurement frequency. For measurements at $100 \mathrm{~Hz}$, the average sensitivity of the sensors is $3.8 \mathrm{pF} / \mathrm{RH} \%$ with a standard error of the mean of $0.42 \mathrm{pF} / \mathrm{RH} \%$. For measurements of $1 \mathrm{kHz}$, the average sensitivity is $2.11 \mathrm{pF} / \mathrm{RH}$
$\%$ and the standard error of the mean is $0.23 \mathrm{pF} / \mathrm{RH} \%$. This then further reduces to 1.185 and $0.85 \mathrm{pF} / \mathrm{RH} \%$ with errors of 0.12 and $0.092 \mathrm{pF} / \mathrm{RH} \%$ for 10 and $100 \mathrm{kHz}$, respectively. A decrease in sensitivity with an increasing frequency is to be expected as data shown in previous work details that the relative permittivity of the capacitor decreases with increasing frequency. ${ }^{20}$ Permittivity is the ability of a material to store electrical energy, and this is dictated by how well a material can be polarized by an electromagnetic wave. With a shorter wavelength at high frequencies, the polarization of the wave is faster than the polarization of the material; therefore, permittivity decreases with an increasing frequency. In the literature, most off-the-shelf capacitive humidity sensors have a typical sensitivity of $<0.5 \mathrm{pF} / \mathrm{RH} \%{ }^{23}$ This means the $4 \mathrm{~cm}^{2}$ capacitive humidity sensors in comparison to the literature on commercial, discrete humidity sensors are classified as high sensitivity alongside being highly linear at higher frequencies. Analyzing the data, although the sensors follow the typical nonlinear pattern, the degree of linearity is exceptionally high for a humidity sensor. Plotting the lines of best fits for each replicate and repeat results in an average $R^{2}$ value of 0.9772 with a standard error of the mean of 0.004822 allowing for the use of a linear approximation in the programming for any device utilizing these printed sensors. The percentage increase in capacitance between 5 and 95\% relative humidity was 204, 164,140 , and $127 \%$ for $100 \mathrm{~Hz}, 1 \mathrm{kHz}, 10 \mathrm{kHz}$, and $100 \mathrm{kHz}$, respectively. This demonstrates that at a lower measurement frequency, the humidity response signal is higher.

For the $9 \mathrm{~cm}^{2}$ humidity sensors, the average sensitivity of the sensors and the standard errors of the mean were calculated for each measurement frequency. As can be seen in Figure S1, for measurements at $100 \mathrm{~Hz}$, the average sensitivity of the sensors is $7.76 \mathrm{pF} / \mathrm{RH} \%$ with a standard error of the mean of $1.04 \mathrm{pF} /$ $\mathrm{RH} \%$. For measurements of $1 \mathrm{kHz}$, the average sensitivity is $3.69 \mathrm{pF} / \mathrm{RH} \%$ and the standard error of the mean is $0.51 \mathrm{pF} /$ $\mathrm{RH} \%$. This then further reduces to 2.76 and $1.93 \mathrm{pF} / \mathrm{RH} \%$ 
Table 1. Comparison of Printed Sensors

\begin{tabular}{|c|c|c|c|c|c|}
\hline material & sensor type & response & recovery & range $(\%)$ & ref \\
\hline carbon nanotubes/HEC & resistive & $20 \mathrm{~s}$ & $35 \mathrm{~s}$ & $20-80$ & 15 \\
\hline graphene oxide & resistive & $2.7 \mathrm{~s}$ & $4.7 \mathrm{~s}$ & $11-97$ & 24 \\
\hline paper & capacitive & $250 \mathrm{~s}$ & $175 \mathrm{~s}$ & $20-90$ & 25 \\
\hline graphene/zinc oxide & impedance & $1 \mathrm{~s}$ & $2 \mathrm{~s}$ & $0-85$ & 16 \\
\hline PDMS-ClC2 & capacitive & $120 \mathrm{~s}$ & $\mathrm{~N} / \mathrm{A}$ & $30-60$ & 14 \\
\hline ITO/alumina $\left(4 \mathrm{~cm}^{2}\right)$ (response/recovery range) & capacitive & $21.4 \mathrm{~s}(13.5-26.5)$ & $4.8 \mathrm{~s}(4-6)$ & $5-95$ & this work \\
\hline ITO/alumina $\left(9 \mathrm{~cm}^{2}\right)$ (response/recovery range) & capacitive & $47.2 \mathrm{~s}(43-56)$ & $49.5 \mathrm{~s}(49-76.5)$ & $5-95$ & this work \\
\hline
\end{tabular}

with errors of 0.28 and $0.018 \mathrm{pF} / \mathrm{RH} \%$ for 10 and $100 \mathrm{kHz}$, respectively. This follows the same trend as $4 \mathrm{~cm}^{2}$ sensors while providing much greater sensitivity. The average linearity for each response curve is an average $R^{2}$ of 0.9738 with a standard error of the mean of 0.002793 . Therefore, although the system has just as good linearity as the $4 \mathrm{~cm}^{2}$ capacitors, it has the added benefit of an even higher sensitivity as shown in Figure 2a, suggesting the sensitivity can be customized by altering the sensor size.

3.2. Humidity Sensor Response. Due to the parallel plate structure of the humidity sensors, there are two routes for water to be absorbed into the dielectric layer to enable capacitive humidity sensing. One route is for the water absorption to occur on the edges of the humidity sensor where, as can be seen in Figure 1a, the dielectric layer is exposed. The second route is where water can be absorbed through the conductive metal oxide layer, something not usually possible with metallic-based conductive inks, which form a highly dense film. As the ITO layer is porous as shown in Figure 1c, water can pass through the layer and into the dielectric material with ease as demonstrated in Figure 2c. Traditionally with parallel plate capacitors, a section of the conductive plate would be removed to allow this, with the consequence of reduced capacitance; however, using the conductive metal oxide ink provides an advantage in that capacitance can be kept high. To avoid this, interdigitated structures are generally used for sensing; however, for printed electronics, this adds complexity to the printing process with performance being dictated by printing resolution and quality. To provide information of how printed humidity sensors in this configuration respond to changes in relative humidity, sensors were measured for the response and recovery times in a controlled temperature (constant $25{ }^{\circ} \mathrm{C}$ ) and humidity chamber. Response and recovery times were measured between four ranges of humidity, these being 15-30, 15-50, 15-70, and 15-90\%. The upper and lower limits of 90 and $15 \%$ were selected due to the chamber taking longer than the sensors' response and recovery times to reach a relative humidity below $15 \%$ or above $90 \%$, eliminating any confounding of data from the experimental error. The response time is calculated by measuring the capacitance and relative humidity with time and is shown in Figure $2 \mathrm{~b}$. Once the humidity reaches its maximum value, the response time is measured as the time taken for the sensor reading to plateau. Again, for the recovery time, it is measured as the time taken for the humidity sensor to plateau once the humidity chamber has reached its targeted humidity. For $4 \mathrm{~cm}^{2}$ sensors, in the range of $15-30 \%$ relative humidity, the response time is $13.5 \mathrm{~s}$ with a recovery time of 4 s. Moving up to a $50 \%$ relative humidity, the response time changes to $26.5 \mathrm{~s}$ and a recovery time of $4 \mathrm{~s}$. At $70 \%$ relative humidity, response times were in the same range as previous measurements at $25.5 \mathrm{~s}$ and the recovery time $6 \mathrm{~s}$. Finally, for measurements at $90 \%$ relative humidity, a peak was reached at $18 \mathrm{~s}$ and the response minimum measured at $4.5 \mathrm{~s}$. To compare with the literature, the weighted average response and recovery times are calculated. Therefore, the average response and recovery times were 21.4 and $4.8 \mathrm{~s}$, respectively. Alumina has been commonly used in various humidity sensors in the literature, however never in a fully printed capacitive humidity sensor before. Analyzing the literature, the structure of the alumina is considered to provide a major contribution to the response and recovery times. Metal oxides sense primarily through diffusion, so they typically have slower response times than some other materials with a range in the observed literature of $28 \mathrm{~s}$ to $>240 \mathrm{~min}$. Therefore, these sensors display faster response times than previously reported work. Any recovery or response time below $<5 \mathrm{~s}$ is considered fast, demonstrating that the sensors have a typical response time but a fast recovery time at $4.8 \mathrm{~s}$, most likely due to the printed films allowing for quick diffusion out of the films.

For the $9 \mathrm{~cm}^{2}$ sensors with a larger area, it is observed that the response and recovery times are longer. As shown in Figure S2, it appears that due to the larger sensor area, a longer time is taken for the dielectric to reach a saturation point where water diffusion is slow. Both response and recovery times are much slower, suggesting a compromise to be considered when altering the sensor size between response and recovery times and then sensitivity depending on the application. For the sensor in the range of $15-30 \%$ relative humidity, the response time is $51.5 \mathrm{~s}$ with a recovery time of $49 \mathrm{~s}$. Moving up to a 50\% relative humidity, the response time changes to $56 \mathrm{~s}$ with a recovery time of 76.5 s. At $70 \%$ relative humidity, response times were in the same range as previous measurements at $45 \mathrm{~s}$ and the recovery time was $68.5 \mathrm{~s}$. Finally, for measurements at $90 \%$ relative humidity, a peak was reached at $43 \mathrm{~s}$ and the response minimum was measured at $72.5 \mathrm{~s}$. Therefore, the weighted average response time of the sensor is $47.2 \mathrm{~s}$ and the recovery time is $49.5 \mathrm{~s}$. The response times measured indicate some sort of linear relationship between the capacitor area and response time, considering the $9 \mathrm{~cm}^{2}$ sensor is $2.25 \mathrm{x}$ larger than the $4 \mathrm{~cm}^{2}$ and the response time is $2.34 \times$ larger. However, the recovery time is much larger than the $4.8 \mathrm{~s}$ of the smaller sensors, suggesting that the water diffusion process out of the film is much slower with an increasing film area. This demonstrates that when designing these sensors for industrial use, tuning the area of the sensor is possible depending on the users' required applications. For fast response applications, smaller sensors can be printed with relatively good sensitivity; however, if higher sensitivity is required, the sensor size can be increased, compromising on the response times. A comparison of the characteristics of this sensor compared with the wider literature of printed humidity sensors can be seen in Table 1 .

Dynamic response was measured by alternating between 60 and $80 \%$ relative humidity and the time between peaks. The 
(a)
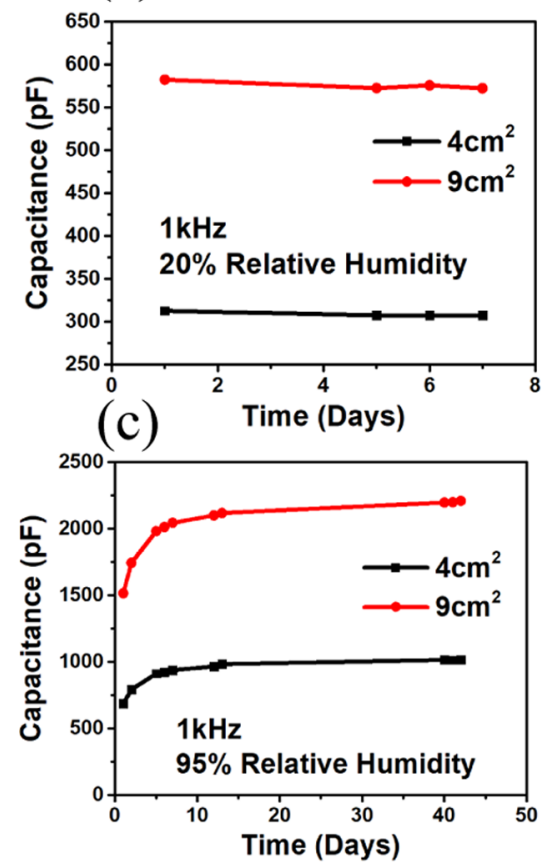

(b)
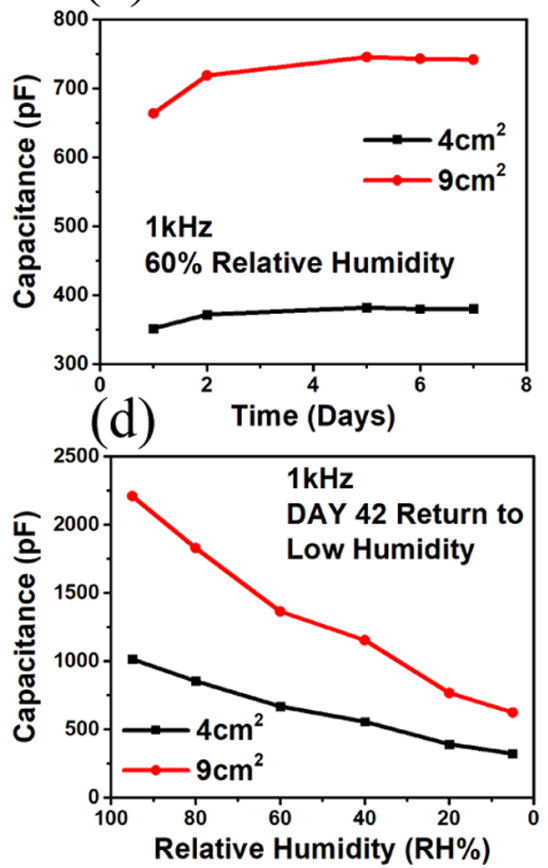

Figure 3. Environmental stability of sensors at (a) $20 \% \mathrm{RH}$, (b) $60 \% \mathrm{RH}$, and (c) $95 \% \mathrm{RH}$ with the (d) return profile from 95 to $5 \%$.

sensors and humidity were measured in real time to provide capacitance and humidity plots, and the data can be seen in Figure $2 \mathrm{~d}$. In the dynamic sensor response, it can be seen that a saturated baseline occurs after the second response. In dynamic response measurements, to account for hysteresis, response and recovery times are calculated as the time taken to move between 10 and $90 \%$ of the baseline and peak capacitance. Calculating the recovery times for the second and third response curves shows a recovery time of $31.5 \mathrm{~s}$ using this method. However, to return to the saturated baseline and to make a full recovery, it takes $241 \mathrm{~s}$. The sensors follow the humidity plot very closely due to their reasonable response and recovery times. An initial observation of hysteresis is made between the first and second peak. However, there is very little hysteresis between the second and third peak, suggesting once an initial saturation point has been reached, due to the delay in diffusion out of the film, the hysteresis becomes minimal as water is only absorbed into the easily accessible parts of the film. To measure the extent of hysteresis, hysteresis loops were measured for both sizes of sensor and can be seen in Figures S3 and S4. For the measurement of hysteresis, due to the use of environmental chamber, the rate of humidity increase is highly controllable; however, the decrease of humidity is not due to an immediate venting of the chamber caused by the control unit. The first measurement, therefore, is a measurement of increasing relative humidity from 5 to $95 \%$; the chamber is then left at $95 \%$ for $10 \mathrm{~min}$ to reach a full saturation, and then decreased in $5 \%$ intervals to $5 \%$ to see how closely the sensors return to their original value. For the 4 $\mathrm{cm}^{2}$ printed sensor hysteresis loops, shown in Figure S3, the final capacitance is generally quite close to the initial starting capacitance. For the sensors at $100 \mathrm{~Hz}$, a $6 \mathrm{pF}$ difference is observed between the start and end of the loop. For 1, 10, and $100 \mathrm{kHz}$, differences of 4.1, 3.2, and $13.5 \mathrm{pF}$ are observed. Percentage hysteresis values that measure the percentage difference in the $\mathrm{Y}$ offset between the positive and negative curves in the hysteresis loops were also calculated. For $100 \mathrm{~Hz}$ the hysteresis percent was calculated to be $35.4 \%$. At the frequencies 1,10 , and $100 \mathrm{kHz}$, hysteresis percentages were found to be $32.5,26.1$, and $28.9 \%$, respectively. For the $9 \mathrm{~cm}^{2}$ sensors, shown in Figure S4, measurements at $100 \mathrm{~Hz}$ show a difference of $82 \mathrm{pF}$, which is much more than the $4 \mathrm{~cm}^{2}$ sensors. This is due to the primary water absorption method being slow diffusion. At $1 \mathrm{kHz}$, the difference between the start and end capacitances is $61.8 \mathrm{pF}$, and for 10 and $100 \mathrm{kHz}$, it is 48.7 and $44.5 \mathrm{pF}$, respectively. Therefore, there is greater hysteresis with respect to a return to the original value in the larger sensors, which due to the diffusion process is to be expected. However, the percentage hysteresis was generally lower in the $9 \mathrm{~cm}^{2}$ sensors. The values calculated for percentage hysteresis in the $9 \mathrm{~cm}^{2}$ capacitors provided a hysteresis percent of $14.7 \%$ at $100 \mathrm{~Hz}$. At the frequencies 1,10 , and $100 \mathrm{kHz}$, hysteresis percentages were found to be 17.1, 17.8 , and $24.1 \%$, respectively. In practical applications, utilizing the dynamic response curves shown in Figure $2 \mathrm{~d}$, this hysteresis can be mitigated for by integrating under the response curve to calculate the humidity, rather than associating a specific capacitance with a specific relative humidity. ${ }^{26}$

3.3. Humidity Sensor Stability. To determine whether hysteresis has a long-term effect on the printed metal oxide humidity sensors, stability measurements were taken at various relative humidity values with the data shown in Figure $3 a-d$.

Stability measurements were taken at three different humidity levels: a low humidity level of $20 \% \mathrm{RH}$, a medium humidity level of $60 \% \mathrm{RH}$, and a high humidity level at $95 \%$ $\mathrm{RH}$. The first measurement was taken an hour after placement into the chamber to allow it to acclimatize to the new humidity. Stability measurements were taken until the readings became stable and can be seen in Figure 3a-c. For both 20 and $60 \% \mathrm{RH}$ experiments, over 7 days, the device gave similar readings with no apparent water damage to the films. With 
regard to the measurements at $20 \% \mathrm{RH}$ for the $4 \mathrm{~cm}^{2}$ sensors, a decrease from 312.7 to $307.4 \mathrm{pF}$ was observed, and for the 9 $\mathrm{cm}^{2}$ sensors, a decrease from 582.5 to $572.5 \mathrm{pF}$ was observed. As this humidity level is lower than the typical environmental humidities, it is possible this is caused by the long-term diffusion of any leftover water in the sensor's premeasurement.

For the sensors measured at $60 \% \mathrm{RH}$, a general increase in capacitance is shown over the first 5 days of measurement before stabilization. The most likely explanation is due to the diffusion of water becoming more difficult after an initial absorption phase, taking days instead of seconds to fully saturate the dielectric film. The initial $4 \mathrm{~cm}^{2}$ capacitance was $351.6 \mathrm{pF}$ with a final stable capacitance of $380.4 \mathrm{pF}$, whereas the $9 \mathrm{~cm}^{2}$ sensor had a capacitance of $664.1 \mathrm{pF}$ moving up to $742.1 \mathrm{pF}$. The $95 \% \mathrm{RH}$ high humidity stability measurements were initially taken for 2 weeks, then left for a further 30 days to account for a full saturation of the dielectric film. The capacitance was found to increase from 686.9 to $1014.6 \mathrm{pF}$ for the $4 \mathrm{~cm}^{2}$ sensor and from 1516.3 to $2211 \mathrm{pF}$ for the $9 \mathrm{~cm}^{2}$ sensor. For the $95 \% \mathrm{RH}$ sensor measurements, to determine whether the high humidity for 45 days had any lasting effects, the humidity was lowered back to $5 \%$ relative humidity with the data shown in Figure 3d. For the $9 \mathrm{~cm}^{2}$ sensor measurements, the sensor moved back to a $5 \% \mathrm{RH}$ of 623.8 $\mathrm{pF}$, whereas the $4 \mathrm{~cm}^{2}$ sensor dropped to $321.9 \mathrm{pF}$. After leaving the sensors in for another $24 \mathrm{~h}$, the final capacitances of the $4 \mathrm{~cm}^{2}$ sensor were 308.1 and $594.5 \mathrm{pF}$ for the $9 \mathrm{~cm}^{2}$, which is exceptionally close to the original values, suggesting that there is no long-term damage from these experiments. This is expected as the metal oxide materials used are generally highly environmentally stable and this stability is a main motivation for fully oxide-based printed electronics, something that typical printed electronics suffers from.

3.4. Humidity Sensing Mechanism Analysis. Impedance spectroscopy is generally the best tool for determining the sensing mechanism of a humidity sensor. The Nyquist plot for the printed metal oxide humidity sensors can be seen in Figure 4. Impedance spectroscopy was performed at 5, 20, 40,

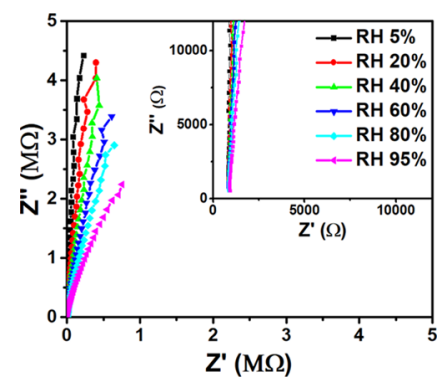

Figure 4. Nyquist plot of sensors at varying relative humidity percentages with a zoom of higher-frequency data inset providing ESR information.

60,80 , and $95 \%$ relative humidity to determine the electronic properties at low and high humidity. From the impedance analysis shown in Figure 4, as the relative humidity increases, the Nyquist plot deviates more and more from an ideal capacitor. A perfect capacitor will have a Nyquist plot with no changing real impedance, and only a change in the imaginary impedance, essentially a $90^{\circ}$ angle to the $x$-axis. The increasing humidity levels raise the amount of ionic conductivity in the film and begin to create a semicircle, which is characteristic of the phenomena. This is also interpreted as the capacitor moving away from being a perfect capacitor and that the water absorption/ionic conductivity is creating a more imperfect capacitor with the water increasing the dissipation factor and the dielectric permittivity (Figure S5) in tandem.

To determine the sensing mechanism from impedance data, an equivalent circuit diagram must be formulated and simulated, which accurately describes the real-world behavior of the sensor, determined by matching the circuit simulation to real-world data. When simulating, knowledge about the design of the electrical system is required. The simulation software used (Autolab Nova v2.0) requires an inputted equivalent circuit diagram to simulate an electronic device. The software then alters the values of each component until a match is found. The inputted equivalent circuit diagrams for sensors at both low and high relative humidity are shown in Figure 5. Enlarged versions of these equivalent circuit diagrams with associated input values can be seen in Figure S6. Before simulating, these equivalent circuits must be formulated based on the design and structure of the sensor. With regard to these printed parallel plate capacitors, the structure is a resistive film of ITO, a resistive dielectric film of alumina where the electric field is generated with polarization, and a second resistive film of ITO. Due to this, the $0 \%$ humidity system, or in this case the $5 \%$ humidity model, can be described in equivalent circuit terms as a resistor connected to a resistor (ITO) and capacitive element in parallel (Alumina) connected to a resistor. Since no capacitor can be ideal, the capacitive element is chosen as a constant-phase element, which models as a capacitor with an ideality $(n)$ between 0.9 and 0.99 , which can be seen in the circuit diagram shown in Figure 5a.

One explanation for the capacitive devices of porous materials such as this system is that each dielectric particleparticle boundary in the dielectric can create an electric field having a capacitive element, with the particle itself also being resistive. Therefore, the dielectric layer can be modeled as an essentially infinite amount of RC circuits, each with its imperfect capacitance. To represent this as one circuit component, the CPE is used. For the sensing mechanism analysis, the sensor is modeled at both 5 and 95\% humidity, the minimum and maximum that can be measured in the experimental setup. We can then observe the system with no water absorption and the system with maximum water absorption. The equivalent circuit diagrams to represent this can be seen in Figure 5a alongside measured and simulated data fitting the measured data. For capacitors, ideality in the capacitor is measured with the coefficient $N$. For the no water absorption system, the circuit matches the 5\% relative humidity with the constant phase element showing a nearperfect capacitor, where $N=0.988$. Adding a second CPE to one of the ITO parts of the equivalent circuit diagram shows the $N$ value of the ITO section at 0.945 and the $N$ value of the Alumina at 0.93 , showing that the capacitance is becoming more imperfect due to water absorption into both films. With these equivalent circuit diagrams, Nyquist and bode plots are simulated. As seen in Figure 5a, the simulation of the 5\% RH equivalent circuit diagram provides a near-perfect fit to the experimental data. However, it does not fit the $95 \% \mathrm{RH}$ data, proving that a new element is required to describe the sensing system. The simulated circuit diagram for the system at $95 \%$ $\mathrm{RH}$, with the CPE added to the ITO part, provides again a near-perfect simulated bode plot and Nyquist plot as shown in Figure $5 \mathrm{~b}$. As can be seen in both the Nyquist and bode plots, the simulated equivalent circuit data provides a good fit. Using 

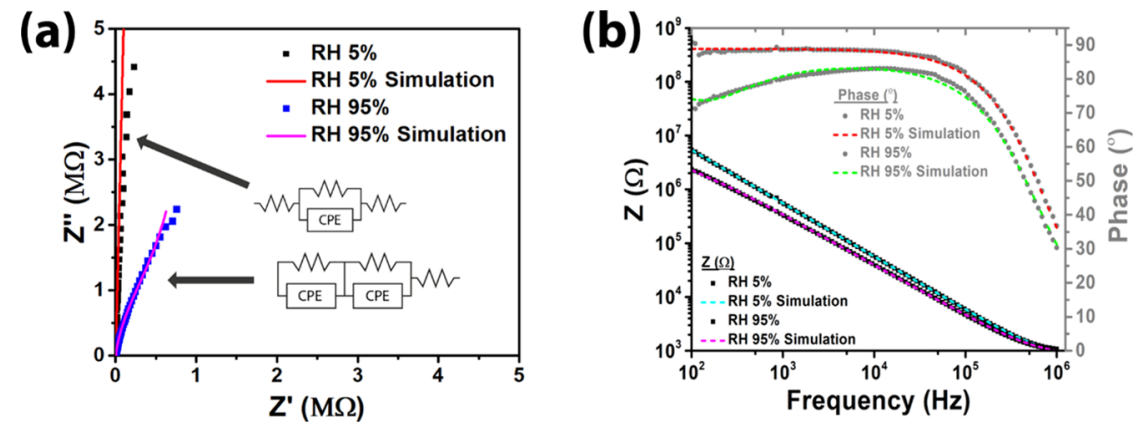

Figure 5. Graph detailing a (a) low- and high-humidity Nyquist plot showing the equivalent circuit diagrams for sensing at different humidity levels and the associated simulations of the circuit diagrams matching the measured data and (b) a bode plot measured at low and high relative humidity with matched simulation data from the equivalent circuit diagram.

the knowledge from the literature on sputter-coated ITO capacitive humidity sensors, it can be assumed that at the $95 \%$ relatively humidity point, the water creates a semicircle, a sign of ionic conductivity in the dielectric that is typical of humidity sensing. ${ }^{19}$ This can be seen in the increase in real impedance (the resistive component) increasing into the order of $M \Omega$, moving closer to the resistance of water than the ITO. Simulations were performed for each impedance measurement to determine at which humidity the indium tin oxide starts contributing; it was found that the ITO electrode provided no contribution to the humidity sensing until $80 \%$ relative humidity. This humidity percentage is the point where the system becomes nonlinear in the humidity versus capacitance data in Figure 2a. This suggests that the mechanism of the humidity sensor is the absorption of water into the alumina pores, increasing the ionic conductivity and the dielectric constant essentially creating a Helmholtz electrical double layer.

Impedance spectroscopy was performed for the samples after stability measurements (Figure $3 \mathrm{~d}$ ) at various relative humidity percentages to gauge how they interact with humidity once saturated. Data for both 4 and $9 \mathrm{~cm}^{2}$ can be seen in Figure S7a,b, which displays a similar trend to Figure 4. Post saturation, the sensors still react to different relative humidity percentages, showing that they still function as humidity sensors. However, the ideality of the capacitors is reduced due to the presence of water in the saturated films. At saturated concentrations of water, the mechanism is unchanged following a similar clear trend.

\section{CONCLUSIONS}

For the first time, a fully metal oxide, printed, parallel plate capacitive humidity sensor was designed and fabricated from conductive ITO inks and dielectric alumina inks, which showed good sensitivity, good response, and fast recovery times. It was found that by altering the size of the sensor its properties can be tailored to the needs of the user. Measurements were undertaken for a wide humidity range (5-95\% RH) and were found to provide highly linear responses. The sensors exhibited good sensitivity in the range of $0.85-7.76 \mathrm{pF} / \mathrm{RH} \%$. For smaller sensors, response times were measured to be $21.4 \mathrm{~s}$ on average and recovery times were $4.8 \mathrm{~s}$ on average. Recovery time increased with a larger sensor size but was accompanied by an increase in sensitivity. This shows that the properties of the sensor can be customized depending on the printed area of the capacitor. Impedance spectroscopy was used to determine the sensing mechanism, which was found to be primarily alumina at lower humidities, with the ITO contributing with increasing the ionic conductivity at higher humidities, contributing to the high linearity. These printed sensors enable future work to be conducted in fully printed environmental monitoring for applications such as smart buildings and smart packaging, using the low-waste, high-throughput screen-printing method.

\section{ASSOCIATED CONTENT}

\section{SI Supporting Information}

The Supporting Information is available free of charge at https://pubs.acs.org/doi/10.1021/acsaelm.0c00660.

Hysteresis loops; capacitance versus frequency data for 9 $\mathrm{cm}^{2}$ sensors; response and recovery versus time data for $9 \mathrm{~cm}^{2}$ sensors; permittivity versus relative humidity percentage data; and sensing mechanism analysis performed at saturation (PDF)

\section{AUTHOR INFORMATION}

\section{Corresponding Author}

Jack R. McGhee - Design Research for Additive Manufacturing Research Lab, Loughborough Design School, Loughborough University, Loughborough LE11 3TU, U.K.; 이잉.org/ 0000-0001-7372-1966; Email: j.mcghee@lboro.ac.uk

\section{Authors}

Jagdeep S. Sagu - Energy Research Laboratory, Department of Chemistry, Loughborough University, Loughborough LE11 3TU, U.K.

Darren J. Southee - Design Research for Additive Manufacturing Research Lab, Loughborough Design School, Loughborough University, Loughborough LE11 3TU, U.K.; (1) orcid.org/0000-0001-5428-8300

Peter. S. A. Evans - Design Research for Additive Manufacturing Research Lab, Loughborough Design School and Energy Research Laboratory, Department of Chemistry, Loughborough University, Loughborough LE11 3TU, U.K.

K. G. Upul Wijayantha - Energy Research Laboratory, Department of Chemistry, Loughborough University,

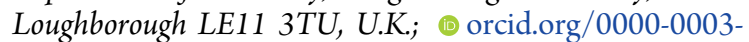
0258-2385

Complete contact information is available at: https://pubs.acs.org/10.1021/acsaelm.0c00660

\section{Author Contributions}

J.R.M. undertook most of the experimental work in this manuscript supported by J.S.S., who aided material character- 
ization and gave intellectual input. D.J.S. provided intellectual input and aided in experimental design and setup. P.S.A.E. provided intellectual input with regard to the ink formulation, and K.G.U.W. provided intellectual input.

\section{Funding}

The research was funded as part of the EPSRC grant (EP/ L017709/1) for research into "Sustainable Manufacturing of Transparent Conducting Oxide (TCO) Inks and Thin Films"-a collaboration between Loughborough University's Design School, the Department of Chemistry, Wolfson School of Mechanical Engineering and Manufacturing and University College London (UCL).

\section{Notes}

The authors declare no competing financial interest.

\section{ACKNOWLEDGMENTS}

The authors greatly appreciate the help of industrial and academic partners. Help was received from Professor Bill MacDonald of Dupont Teijin Films, who has supplied the PET films used. The authors acknowledge use of facilities within the Loughborough Materials Characterization Centre.

\section{ABBREVIATIONS}

ITO, indium tin oxide; NC, nitrocellulose

\section{REFERENCES}

(1) Chen, Z.; Lu, C. Humidity Sensors: A Review of Materials and Mechanisms. Sens. Lett. 2005, 3, 274-295.

(2) Farahani, H.; Wagiran, R.; Hamidon, M. Humidity Sensors Principle, Mechanism, and Fabrication Technologies: A Comprehensive Review. Sensors 2014, 14, 7881-7939.

(3) Ibrahim, M.; Elgamri, M.; Babiker, A.; Mohamed, A. In Internet of Things based Smart Environmental Monitoring Using the Raspberry $P i$ Computer, Fifth International Conference on Digital Information Processing and Communications (ICDIPC), 2015.

(4) Chieochan, O.; Saokaew, A.; Boonchieng, E. In IOT for Smart Farm: A Case Study of the Lingzhi Mushroom Farm at Maejo University, International Joint Conference on Computer Science and Software Engineering (JCSSE), 2017.

(5) Al-Kuwari, M.; Ramadan, A.; Ismael, Y.; Al-Sughair, L.; Gastli, A.; Benammar, M. In Smart-Home Automation Using IoT-Based Sensing and Monitoring Platform, IEEE 12th International Conference on Compatibility, Power Electronics and Power Engineering (CPEPOWERENG 2018), 2018.

(6) Karim, A.; Hassan, A.; Akanda, M. Monitoring food storage humidity and temperature data using IoT. Food Process. Technol. 2018, 6, 400-404.

(7) Navratil, J.; Rericha, T.; Soukup, R.; Hamacek, A. In Aerosol Jet Printed Sensor on Fibre for Smart and IoT Applications, 41st International Spring Seminar on Electronics Technology (ISSE), 2018.

(8) Tentzeris, M.; Kim, S.; Vyas, R.; Traille, A.; Pons, P.; Aubert, H.; Georgiadis, A.; Collado, A. In Inkjet-Printed "Zero-Power" Wireless Sensor and Power Management Nodes for IoT and "Smart Skin" Applications, 20th International Conference on Microwaves, Radar and Wireless Communications (MIKON), 2014.

(9) Virtanen, J.; Ukkonen, L.; Bjorninen, T.; Elsherbeni, A.; Sydanheimo, L. Inkjet-Printed Humidity Sensor for Passive UHF RFID Systems. IEEE Trans. Instrum. Meas. 2011, 60, 2768-2777.

(10) Ali, S.; Hassan, A.; Hassan, G.; Bae, J.; Lee, C. All-printed humidity sensor based on graphene/methyl-red composite with high sensitivity. Carbon 2016, 105, 23-32.

(11) Hay, G. I.; Evans, P.; Harrison, D.; Southee, D.; Simpson, G.; Harrey, P. Characterisation of lithographically printed resistive strain gauges. IEEE Sens. J. 2005, 5, 864-871.
(12) Kim, J.; Kim, W. A paired stretchable printed sensor system for ambulatory blood pressure monitoring. Sens. Actuators, A 2016, 238, 329-336.

(13) Chen, K.; Gao, W.; Emaminejad, S.; Kiriya, D.; Ota, H.; Nyein, H.; Takei, K.; Javey, A. Printed Carbon Nanotube Electronics and Sensor Systems. Adv. Mater. 2016, 28, 4397-4414.

(14) Komazaki, Y.; Uemura, S. Stretchable, printable, and tunable PDMS-CaCl2 microcomposite for capacitive humidity sensors on textiles. Sens. Actuators, B 2019, 297, No. 126711.

(15) Turkani, V. S.; Maddipatla, D.; Narakathu, B.; Saeed, T.; Obare, S.; Bazuin, B.; Atashbar, M. A highly sensitive printed humidity sensor based on a functionalized MWCNT/HEC composite for flexible electronics application. Nanoscale Adv. 2019, 2311.

(16) Hassan, G.; Lee, C.; Hassan, A. Wide range and stable ink-jet printed humidity sensor based on graphene and zinc oxide nanocomposite. J. Mater. Sci.: Mater. Electron. 2018, 29, 5806-5813.

(17) Wang, C.; Yin, L.; Zhang, L.; Xiang, D.; Gao, R. Metal oxide gas sensors: Sensitivity and influencing factors. Sensors 2010, 10, 20882106.

(18) Seiyama, T.; Yamazoe, N.; Arai, H. Ceramic Humidity Sensors. Sens. Actuators 1983, 4, 85-96.

(19) McGhee, J.; Sagu, J.; Southee, D.; Wijayantha, U. Humidity Sensing Properties of Transparent Sputter-Coated Indium-Tin Oxide and Printed Polymer Structures. IEEE Sens. J. 2018, 18, 7358-7364.

(20) McGhee, J.; Southee, D.; Evans, P.; Middlemiss, R.; Wijayantha, U. In Flexible, All Metal Oxide Capacitors for Printed Electronics, 13th IEEE Nanotechnology Materials and Devices Conference, Portland, OR, 2018.

(21) McGhee, J.; Goulas, A.; Southee, D.; Sagu, J.; Engstrom, D.; Wang, J.; Hutt, D.; Evans, P.; Zhou, Z.; Wijayantha, K.; Conway, P.; Carmalt, C. Indium tin oxide nanowires manufactured via printing and laser irradiation. Appl. Mater. Today 2020, 21, No. 100835.

(22) Zhang, S.; Arya, R.; Pandey, S.; Vardaxoglou, J.; Whittow, W.; Mittra, R. 3D-printed planar graded index lenses. IET Microwaves Antennas Propag. 2016, 10, 1411-1419.

(23) Juhász, L.; Mizsei, J. Humidity sensor structures with thin film porous alumina for on-chip integration. Thin Solid Films 2009, 517, 6198-6201.

(24) He, P.; Brent, J.; Ding, H.; Yang, J.; Lewis, D.; P. O’Brien, P.; B. Derby, B. Fully printed high performance humidity sensors based on two-dimensional materials. Nanoscale 2018, 10, 5599-5606.

(25) Gaspar, C.; Olkkonen, J.; Passoja, S.; Smolander, M. Paper as Active Layer in Inkjet-Printed Capacitive Humidity Sensors. Sensors 2017, 17, 1464.

(26) Hay, G.; Southee, D.; Evans, P.; Simpson, G.; Wood, J. A time-strain monitoring system fabricated via offset lithographic printing. Smart Mater. Struct. 2008, 18, No. 015015. 